\title{
Netupitant/palonosetron (NEPA) and dexamethasone for prevention of emesis in breast cancer patients receiving adjuvant anthracycline plus cyclophosphamide: a multi-cycle, phase II study
}

Roberta Caputo ${ }^{1}$, Marina Elena Cazzaniga ${ }^{2}$, Andrea Sbrana ${ }^{3}$, Rosalba Torrisi ${ }^{4}$, Ida Paris ${ }^{5}$, Monica Giordano ${ }^{6}$, Vincenzo Montesarchio ${ }^{7}$, Valentina Guarneri ${ }^{8,9}$, Laura Amaducci ${ }^{10}$, Domenico Bilancia ${ }^{11}$, Giuseppina Cilenti ${ }^{12}$, Alessandra Fabi ${ }^{13}$, Elena Collovà ${ }^{14}$, Alessio Schirone ${ }^{15}$, Erminio Bonizzoni ${ }^{16}$, Luigi Celio ${ }^{17}$, Sabino De Placido ${ }^{18}$ and Michelino De Laurentiis ${ }^{1 *}$

\begin{abstract}
Background: NEPA is an oral fixed-dose combination of netupitant, a new highly selective neurokinin-1 receptor antagonist, and palonosetron. This study was conducted to evaluate whether the efficacy of NEPA against chemotherapy-induced nausea and vomiting (CINV) in cycle 1 would be maintained over subsequent chemotherapy cycles in breast cancer patients receiving adjuvant anthracycline plus cyclophosphamide (AC). The study also describes the relationship between efficacy on day 1 through 5 (overall period) and control of CINV on day 6 through 21 (very late period) in each cycle.

Methods: In this multicentre, phase II study, patients received both NEPA and dexamethasone (12 mg intravenously) just before chemotherapy. The primary efficacy endpoint was overall complete response (CR; no emesis and no rescue medication use) in cycle 1. Sustained efficacy was evaluated during the subsequent cycles by calculating the rate of CR in cycles 2-4 and by assessing the probability of sustained CR over multiple cycles. The impact of both overall CR and risk factors for CINV on the control of very late events (vomiting and moderate-tosevere nausea) were also examined.

(Continued on next page)
\end{abstract}

\footnotetext{
* Correspondence: m.delaurentiis@istitutotumori.na.it

'Breast Medical Oncology Division, Istituto Nazionale Tumori IRCCS

"Fondazione G. Pascale", Naples, Italy

Full list of author information is available at the end of the article
}

(c) The Author(s). 2020 Open Access This article is licensed under a Creative Commons Attribution 4.0 International License, which permits use, sharing, adaptation, distribution and reproduction in any medium or format, as long as you give appropriate credit to the original author(s) and the source, provide a link to the Creative Commons licence, and indicate if changes were made. The images or other third party material in this article are included in the article's Creative Commons licence, unless indicated otherwise in a credit line to the material. If material is not included in the article's Creative Commons licence and your intended use is not permitted by statutory regulation or exceeds the permitted use, you will need to obtain permission directly from the copyright holder. To view a copy of this licence, visit http://creativecommons.org/licenses/by/4.0/. The Creative Commons Public Domain Dedication waiver (http://creativecommons.org/publicdomain/zero/1.0/) applies to the data made available in this article, unless otherwise stated in a credit line to the data. 
(Continued from previous page)

Results: Of the 149 patients enrolled in the study, 139 were evaluable for a total of 552 cycles; $97.8 \%$ completed all 4 cycles. The proportion of patients with an overall CR was $70.5 \%$ (90\% Cl, 64.1 to 76.9$)$ in cycle 1, and this was maintained in subsequent cycles. The cumulative percentage of patients with a sustained CR over 4 cycles was 53\%. NEPA was well tolerated across cycles. In each cycle, patients with CR experienced a significantly better control of very late CINV events than those who experienced no CR. Among the patients with CR, the only predictor for increased likelihood of developing very late CINV was pre-chemotherapy (anticipatory) nausea (adjusted odds ratio $=0.65-0.50$ for no CINV events on cycles 3 and 4).

Conclusion: The high anti-emetic efficacy seen with the NEPA regimen in the first cycle was maintained over multiple cycles of adjuvant AC for breast cancer. Preliminary evidence also suggests that patients achieving a CR during the overall period gain high protection even against very late CINV events in each chemotherapy cycle.

Trial registration: This trial was retrospectively registered at Clinicaltrials.gov identifier (NCT03862144) on 05/Mar/ 2019.

Keywords: NEPA, CINV, Nausea, Vomiting, Breast cancer, AC

\section{Background}

Cytotoxic chemotherapy remains an essential component for the management of breast cancer patients. Both gender and younger age affect the intrinsic emetogenicity of the chemotherapy regimen that still remains as the most important risk factor for chemotherapyinduced nausea and vomiting (CINV) [1]. Indeed, women have an increased risk of CINV, and younger patients (less than 50 years of age) are also more likely than older patients to develop CINV [1]. The combination of an anthracycline and cyclophosphamide $(\mathrm{AC})$ is the backbone of the most effective adjuvant regimens for high-risk early-stage breast cancer. Although anthracyclines and cyclophosphamide are individually considered as being moderately emetogenic, it has been recognized that women receiving the combination of $\mathrm{AC}$ are at a particularly high risk of CINV [2]. Accordingly, the combination of $\mathrm{AC}$ is now classified as highly emetogenic chemotherapy (HEC) by international guidelines [3, 4]. The recommended anti-emetic prophylaxis consists of a triple regimen containing a 5-hydroxytryptamine-3 receptor antagonist (5- $\left.\mathrm{HT}_{3} \mathrm{RA}\right)$, a neurokinin-1 receptor antagonist (NK-1RA), and single-dose dexamethasone. This regimen can help to control nausea and vomiting over the 5-day period of highest emetic risk after chemotherapy administration $[4,5]$. It is also important to underline that successful CINV prevention in the first cycle of therapy should be sustained throughout all planned chemotherapy cycles [6].

Netupitant is a highly selective NK-1RA that exhibits a plasma half-life of approximately $96 \mathrm{~h}$, making singleprophylaxis dosing appropriate [7]. Palonosetron is a pharmacologically distinct $5-\mathrm{HT}_{3} \mathrm{RA}$ that demonstrates prolonged inhibition of $5-\mathrm{HT}_{3}$ receptor function and inhibits $5-\mathrm{HT}_{3}-\mathrm{NK}-1$ receptor crosstalk $[8,9]$. It should be noted that in breast cancer patients receiving $\mathrm{AC}$ anti-emetic guidelines updated from the Multinational Association of
Supportive Care in Cancer recommend palonosetron as the preferred $5-\mathrm{HT}_{3} \mathrm{RA}$, when an NK-1RA is not available [4]. Synergy of netupitant with palonosetron has been demonstrated in vitro, suggesting the potential for an improved efficacy of this combination in clinical practice [8]. NEPA is an oral, single-dose, single-capsule, fixed-combination antiemetic drug containing netupitant and palonosetron that is able to target the two major pathways involved in the transmission of emetic stimuli to the central nervous system during the acute and delayed phases of CINV [7]. In light of this, NEPA has the potential not only to simplify antiemetic coverage but also improve guideline adherence in clinical practice with a convenient, single oral dose. The efficacy and safety of NEPA have been demonstrated in randomised trials involving chemotherapy-naive patients predominantly affected by solid tumors [7]. A pivotal trial in breast cancer patients treated with AC-containing chemotherapy demonstrated superior efficacy of a single dose of NEPA plus dexamethasone for CINV prevention, when compared with palonosetron plus single-dose dexamethasone in cycle 1 of therapy [10]. Studies have also shown NEPA to be well-tolerated over multiple cycles of emetogenic chemotherapy regimens [7]. It is important to highlight that a double-blind study in healthy subjects showed that administration of NEPA caused no significant effects on cardiac function, even at supra-therapeutic doses [11]. This is a very important issue especially in patients who are receiving chemotherapy containing potentially cardio-toxic agents such as the anthracyclines.

On the basis of this evidence, we decided to challenge NEPA efficacy and safety in a clinical setting where patients receive multiple cycles of the same chemotherapy regimen and anti-emetics should demonstrate a sustained benefit over all planned chemotherapy cycles. This phase II study was designed to evaluate whether the anti-emetic efficacy of NEPA plus single-dose dexamethasone observed in cycle 1 would be maintained 
over subsequent cycles of $\mathrm{AC}$ in patients with earlystage breast cancer. In addition, this study describes the relationship between anti-emetic efficacy in the overall study period ( 5 days after chemotherapy administration) and control of symptoms over the very late period (day 6 through 21 of a cycle) in a challenging setting of CINV.

\section{Methods}

\section{Study design}

This was a prospective, multicentre, single-arm, phase II study evaluating NEPA over four consecutive cycles of adjuvant chemotherapy including the combination of AC (doxorubicin or epirubicin plus cyclophosphamide) in breast cancer patients. The study was conducted in accordance with the Good Clinical Practice guidelines, at 22 Italian centers, which were coordinated by the GIM (Gruppo Italiano Mammella) cooperative group, from May to September 2016. Institutional ethics approval was granted at each participating center and written informed consent was obtained from each patient before enrolment.

\section{Patients}

Female patients aged 18 years or over scheduled to receive AC-containing chemotherapy regimen for the adjuvant treatment of invasive breast carcinoma were eligible to participate. Patients were required to have an Eastern Cooperative Oncology Group (ECOG) performance status of 0,1 , or 2 . Women of childbearing potential were also required to use reliable contraceptive measures during the study treatment. Patients had to be without episodes of emesis for $24 \mathrm{~h}$ before study entry, and no emesis because of any organic cause before study entry. Adequate hepatic and renal functions were required. Exclusion criteria included myocardial infarction within 6 months before study entry, uncontrolled diabetes mellitus, concurrent use of any drug with known anti-emetic efficacy, or presence of psychiatric or brain disorders that might interfere with ability to comply with study protocol.

\section{Interventions}

The chemotherapy consisted of either doxorubicin intravenously (iv) $\left(60 \mathrm{mg} / \mathrm{m}^{2}\right)$ or epirubicin iv $\left(90 \mathrm{mg} / \mathrm{m}^{2}\right)$, each administered in combination with cyclophosphamide $\left(600 \mathrm{mg} / \mathrm{m}^{2}\right)$ iv on day 1 of a 21 -day cycle. For all patients, anti-emetic coverage consisted of oral NEPA (netupitant $300 \mathrm{mg} /$ palonosetron $0.50 \mathrm{mg}$ ) plus a single intravenous dose of dexamethasone $12 \mathrm{mg}$, both given before the administration of each chemotherapy cycle. NEPA was administered approximately $60 \mathrm{~min}$ before the start of chemotherapy on day 1 . The use of rescue medications (metoclopramide and/or dexamethasone) for treatment of nausea and/or vomiting occurring within the 5 days after chemotherapy administration was considered treatment failure.

\section{Study outcomes}

The primary efficacy end point of this study was CR (defined as no emesis, and no use of rescue medication) during the overall study period (day 1 through 5 post-chemotherapy). Secondary end points were the proportion of patients who achieved the following during the overall, acute $(0-24 \mathrm{~h}$ post-chemotherapy), and delayed (day 2 through 5 postchemotherapy) periods: CR (not including overall period), and no clinically significant nausea (defined as none to mild in severity). Nausea was graded daily using a four-point categorical Likert scale ( 0 , none; 1 , mild; 2 , moderate; 3 , severe), according to subjective assessment by each patient. All study patients were asked to complete a patient's diary on a daily basis for the overall observation period (from the start of chemotherapy infusion on day 1 through the morning of day 6 of each cycle). Patients recorded daily any emetic episode and rescue medication intake as well as prechemotherapy experience (just before chemotherapy initiation) of either nausea or anxiety on a 11-point numerical rating scale (NRS; with 10 being the most severe) for each cycle. Pre-chemotherapy nausea and anxiety were defined a priori as a score of 1 or greater, and clinically significant prechemotherapy nausea and anxiety were defined as a score of 3 or greater [12]. At the end of the overall study period, each patient continued to complete the diary on a daily basis in order to capture any episodes of vomiting and/or nausea during the very late observation period (day 6 through 21) for each chemotherapy cycle. An exploratory efficacy end point was the proportion of patients without CINV events (vomiting and moderate-to-severe nausea) during the very late observation period in each cycle. Safety was assessed by treatment-emergent adverse events (TEAEs). Any serious TEAE judged by the investigator to be possibly, probably, or definitely related to the study treatment was recorded and graded according to the common terminology criteria for adverse events (CTCAE), version 4.3.

\section{Statistical analysis}

The primary aim of this study was to evaluate the antiemetic efficacy of NEPA plus single-dose dexamethasone based on the proportion of patients with a $\mathrm{CR}$ during the overall period of each cycle of AC-containing chemotherapy. The study was planned according to a one-stage Fleming design with a total sample size of 135 evaluable patients to decide whether the proportion of CR was $>64 \%$ (maximum response proportion of a poor anti-emetic regimen) during the overall period, with a type I error of $5 \%$ (onesided) and type II error of $20 \%$ assuming a minimum response proportion of a good anti-emetic regimen equal to $74 \%$. Considering an attrition of up to $10 \%, 150$ patients 
were needed at baseline. In this study, the efficacy hypothesis was verified by resorting to two-sided confidence intervals (CIs) with 90\% coverage, that were calculated using the Wald method. Therefore, the null hypothesis was rejected if the lower boundary of the $90 \% \mathrm{CI}$ of the proportion of responders was greater than $64 \%$. In spite of greater emphasis was on efficacy analysis in cycle 1 , the study findings had to be confirmed also in the treatment cycles 2,3 , and 4 . The efficacy analysis population was defined as all patients who received protocol required $\mathrm{AC}$, study treatment and completed the patient's diary in cycle 1 . The safety analysis population consisted of all patients who received chemotherapy and study treatment.

An analysis of sustained overall (days 1 to 5) CR evaluated the probability that a patient would remain a responder over the subsequent cycles of chemotherapy. To accomplish this, a Kaplan-Meier curve was computed with patients who did not sustain a response, considered as treatment failures [13]. In post hoc analyses, we examined the association between overall $\mathrm{CR}$ and very late CINV events within each cycle, with comparison made using Fisher's exact test. We also performed an exploratory analysis to examine the impact of risk factors for CINV such as age, motion sickness, pregnancy-related morning sickness, alcohol intake, and the pre-chemotherapy nausea and anxiety NRS at each cycle on very late CINV using logistic regression models. The analysis was restricted to patients who experienced a CR, and analyses were repeated for each cycle. All analyses were conducted using SAS version 9.4 (SAS Institute, Cary, NC, USA).

\section{Results}

A total of 149 consecutive patients were enrolled in the study, and represent the safety population. Ten patients were excluded from the efficacy analysis $(n=3$ patients withdrew the consent before starting the study, and $n=7$ patients were not evaluable for anti-emetic efficacy), leaving 139 patients in the efficacy population. In this population, a total of 552 chemotherapy cycles were administered, and $97.8 \%$ of the patients completed the four planned cycles.

Baseline patient characteristics including emetic risk factors are shown in Table 1 . The median age was 48 years, and the vast majority of the patients (94\%) were treated with the double chemotherapy regimen containing an anthracycline (27\% doxorubicin and $67 \%$ epirubicin) and cyclophosphamide.

\section{Efficacy during the acute, delayed, and overall study periods}

The CR rates during the acute, delayed, and overall study periods are shown in Table 2. The proportion of patients with an overall CR was $70.5 \%$ (90\% CI, 64.1 to 76.9 ) in cycle 1 , and this was maintained in subsequent cycles. In each cycle, the primary end point was met because the
Table 1 Patient's demographic and clinical characteristics (enrolled population, $n=149$ )

\begin{tabular}{|c|c|c|}
\hline Characteristic & & $\begin{array}{l}\text { NEPA plus } \\
\text { 1-day Dex } \\
\text { No. (\%) }\end{array}$ \\
\hline \multicolumn{3}{|l|}{ Age (years) } \\
\hline Median & 48 & \\
\hline $\min -\max$ & $25-76$ & \\
\hline Age $<50$ years & & $82(55)$ \\
\hline \multicolumn{3}{|l|}{ Height (cm) } \\
\hline Mean & 162.2 & \\
\hline SD & 6.4 & \\
\hline \multicolumn{3}{|l|}{ Weight (kg) } \\
\hline Mean & 66.9 & \\
\hline SD & 14.5 & \\
\hline \multicolumn{3}{|l|}{ ECOG performance status } \\
\hline 0 & & $146(98)$ \\
\hline 1 & & $3(2.0)$ \\
\hline \multicolumn{3}{|l|}{ Chemotherapy regimen } \\
\hline$A C$ & & $40(26.8)$ \\
\hline EC & & $100(67.1)$ \\
\hline FEC & & $5(3.4)$ \\
\hline Other/missing & & $4(2.7)$ \\
\hline \multicolumn{3}{|l|}{ Alcohol consumption } \\
\hline No & & $88(59.1)$ \\
\hline Occasionally ${ }^{a}$ & & $42(28.2)$ \\
\hline Regularly & & $16(10.7)$ \\
\hline Missing & & $3(2.0)$ \\
\hline History of motion sickness & & $47(31.5)$ \\
\hline History of pregnancy-related morning sickness & & $57(38.3)$ \\
\hline
\end{tabular}

NEPA fixed-dose combination of netupitant and palonosetron, Dex dexamethasone, $S D$ standard deviationm, $A C$ anthracycline (i.e., doxorubicin) and cyclophosphamide, EC epirubicin and cyclophosphamide, FEC fluorouracil, epirubicin, and cyclophosphamide

a It is defined as drinking one or two glasses per week

lower limit of $90 \% \mathrm{CI}$ always exceeded the preset cut-off of $64 \%$. CR rates were similar across chemotherapy cycles during the acute and delayed periods. The percentage of patients who experienced a CR in cycle 1 and who sustained a CR over cycles $2-4$ is shown in Fig. 1. The Kaplan-Meier curve showed that more than $50 \%$ of patients sustained a CR over cycles $2-4$. No significant nausea rates were similar across cycles during the delayed and overall periods (Table 2 ).

\section{Incidence of pre-chemotherapy nausea and anxiety}

The incidence of pre-chemotherapy (anticipatory) nausea (score of $\geq 1$ ) increased overall from cycle 1 to cycle 4 , while the incidence of significant pre-chemotherapy nausea (score of $\geq 3$ ) increased only from cycle 1 to cycle 
Table 2 Efficacy end-point analysis (efficacy set population) in patients receiving a single dose of NEPA and dexamethasone

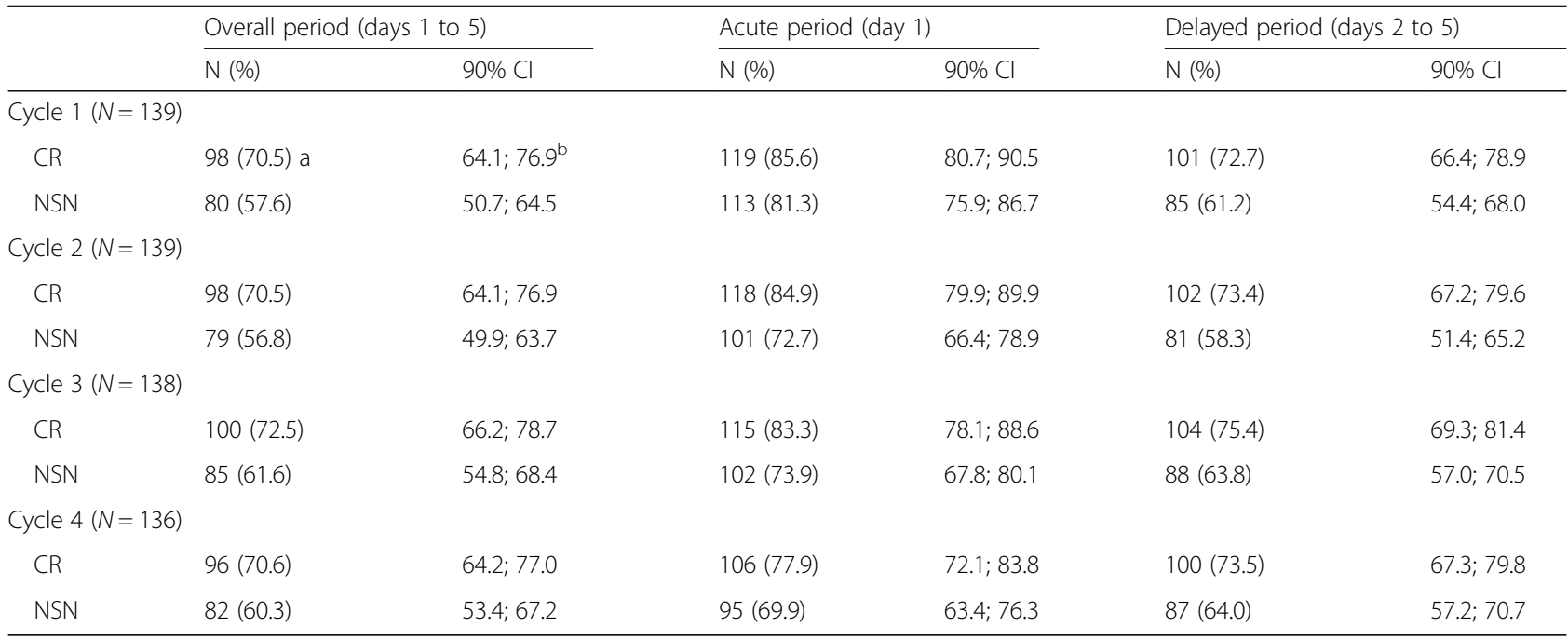

NEPA netupitant plus palonosetron, $C l$ confidence interval, $C R$ complete response (no vomiting and no use of rescue medication), NSN no clinically significant nausea (none to mild in severity)

aPrimary efficacy end point

${ }^{b}$ Efficacy hypothesis was demonstrated as the lower boundary of the $90 \% \mathrm{Cl}$ was greater than the preset cut-off of $64 \%$ which was assumed as the maximum response rate for a poor anti-emetic treatment in the study protocol

3 (Table 3). The intensity of pre-chemotherapy nausea increased over each subsequent cycle, with intensity being almost doubled by cycle 3 .

The incidence of pre-chemotherapy anxiety and significant pre-chemotherapy anxiety decreased across cycles (Table 3). Likewise, the intensity of pre-chemotherapy anxiety decreased across cycles.

\section{Efficacy during the very late period}

The incidence of CINV events (i.e., vomiting and/or moderate-to-severe nausea) during the very late period increased over subsequent cycles $(10.8 \%$ in cycle $1,15.8 \%$ in

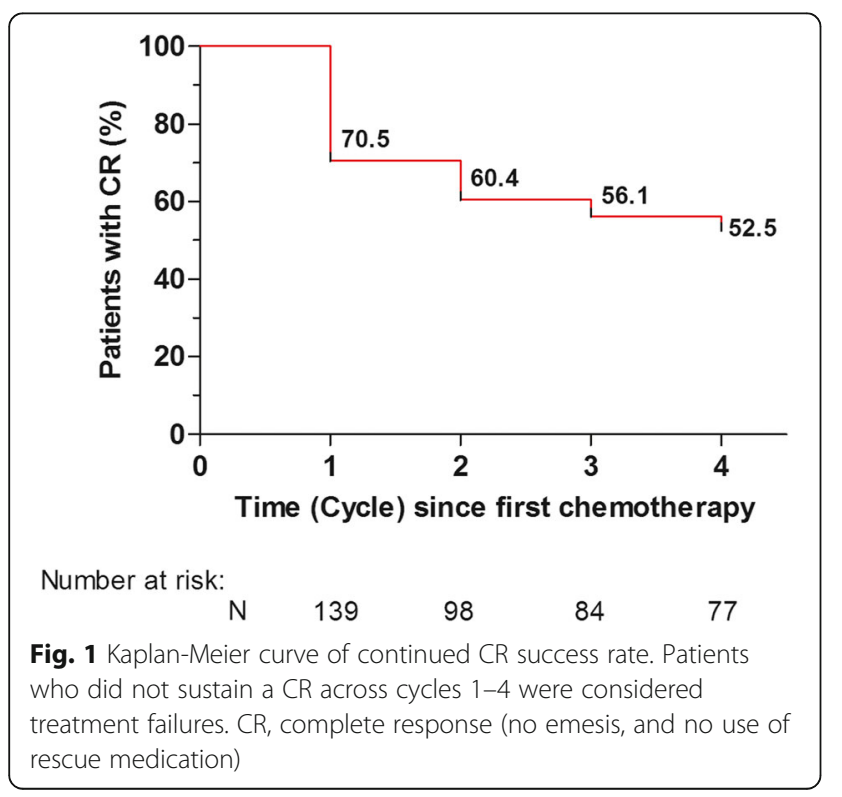

cycle 2, 21\% in cycle 3, and $19.1 \%$ in cycle 4). Overall, five patients experienced both nausea and vomiting, and further two patients had only vomiting in the very late period. In an exploratory analysis, the achievement of a CR during the overall study period was associated with a significantly better control of very late CINV over all chemotherapy cycles (Fig. 2).

A number of patient-related risk factors for CINV were included in a multivariable analysis to assess potential predictors for very late CINV among the patients with $C R$ (Table 4). In this subgroup, the only predictor for increased likelihood of developing very late CINV events was prechemotherapy nausea. However, in cycles 1 and 2, no independent variable was found statistically significant. Conversely, in the third and fourth cycle, the occurrence of nausea ( $\geq 1$ NRS) just before chemotherapy administration emerged as an independent predictor for very late CINV.

\section{Safety}

A total of 146 patients were evaluable for safety in the study. Overall, NEPA was well tolerated over multiple cycles of AC chemotherapy without evidence for increasing adverse events (AEs) across cycles. In this study, the most common treatment-related AEs were fatigue (3.4\%) and headache $(2.1 \%)$. No patient discontinued treatment due to AEs, and no unexpected serious AEs occurred that could be attributed to the anti-emetic regimen across cycles.

\section{Discussion}

The combination of AC is among standard chemotherapy regimens for the treatment of early breast cancer patients, and is now classified as HEC $[4,5]$. In the present 
Table 3 Descriptive summary of pre-chemotherapy nausea and anxiety by chemotherapy cycle

\begin{tabular}{|c|c|c|c|c|c|c|c|c|}
\hline \multirow[b]{2}{*}{ Mean score for prechemotherapy nausea, (0-10 NRS) } & \multicolumn{2}{|c|}{ Cycle $1(\boldsymbol{n}=139)$} & \multicolumn{2}{|c|}{ Cycle $2(\boldsymbol{n}=139)$} & \multicolumn{2}{|c|}{ Cycle $3(\boldsymbol{n}=138)$} & \multicolumn{2}{|c|}{ Cycle $4(\boldsymbol{n}=136)$} \\
\hline & 0.85 & $0.56 ; 1.13$ & 1.47 & $1.08 ; 1.85$ & 1.64 & $1.26 ; 2.01$ & 1.65 & $1.27 ; 2.02$ \\
\hline Pre-chemotherapy nausea ( $\geq 1$ NRS) & $29(20.9 \%)$ & $15.8 ; 27.1$ & $48(34.5 \%)$ & $28.2 ; 41.4$ & $51(36.9 \%)$ & $30.5 ; 43.9$ & $55(40.4 \%)$ & $33.8 ; 47.5$ \\
\hline Significant prechemotherapy nausea ( $\geq 3$ NRS) & $19(13.7 \%)$ & $9.5 ; 19.2$ & $25(17.9 \%)$ & $13.2 ; 23.9$ & $37(26.8 \%)$ & $21.1 ; 33.4$ & $34(25 \%)$ & $19.4 ; 31.6$ \\
\hline Mean score for prechemotherapy anxiety, (0-10 NRS) & 2.68 & $2.25 ; 3.10$ & 1.81 & $1.46 ; 2.16$ & 1.97 & $1.62 ; 2.33$ & 2.03 & $1.66 ; 2.40$ \\
\hline Pre-chemotherapy anxiety ( $\geq 1$ NRS) & $85(61.2 \%)$ & $54.2 ; 67.7$ & $66(47.5 \%)$ & $40.6 ; 54.4$ & $74(53.6 \%)$ & $46.6 ; 60.5$ & $72(52.9 \%)$ & $45.9 ; 59.9$ \\
\hline Significant prechemotherapy anxiety ( $\geq 3$ NRS) & 58 (41.7\%) & $35.1 ; 48.7$ & $40(28.8 \%)$ & $22.9 ; 35.5$ & $43(31.2 \%)$ & $25.1 ; 37.9$ & $48(35.3 \%)$ & $28.9 ; 42.3$ \\
\hline
\end{tabular}

$\mathrm{Cl}$ confidence interval, NRS numerical rating scale (with 10 being the most severe)

Data are reported with $90 \% \mathrm{Cl}$

study, we selected women with early breast cancer receiving $\mathrm{AC}$ as adjuvant chemotherapy. It is important to underline that the majority of patients $(55 \%)$ evaluated in this study was younger than 50 years of age, $32 \%$ had a history of motion sickness, and $38 \%$ of the patients had a history of pregnancy-related morning sickness. All these factors are known to be associated with a higher risk of developing CINV $[14,15]$. Therefore, our patient population can be considered at particularly high risk for CINV.

NEPA, a combination of the new NK-1RA, netupitant and palonosetron, has been designed to improve guideline adherence by packaging guideline-recommended agents in a single oral fixed-dose. Since cancer patients usually receive multiple cycles of chemotherapy, this prospective, phase II study was conducted to evaluate whether the anti-emetic efficacy of NEPA would be maintained over subsequent cycles of AC. The prophylaxis with NEPA plus single-dose dexamethasone resulted in a CR rate of $71 \%$ during the overall study period in cycle 1 , and was maintained through cycle 4 . It is well known that the development of CINV in the first cycle of chemotherapy is a strong predictor of CINV in subsequent cycles [6]. In light of this, it is encouraging that the percentage of patients who experienced a CR in cycle 1 and who sustained a CR over cycles 2-4 was $53 \%$. Interestingly, in a randomised pivotal trial of patients treated with $\mathrm{AC}$, a triple regimen consisting of ondansetron, dexamethasone, and 3-day aprepitant resulted in a CR rate of $51 \%$ during the overall study period in cycle 1 , while $35 \%$ of the patients sustained a CR over chemotherapy cycles 2-4 [13]. It is important to underline that the efficacy of NEPA plus single-dose dexamethasone observed in this study was generally in line with that observed in a recently published pivotal study evaluating the efficacy of the same anti-emetic regimen over multiple cycles of $\mathrm{AC}$ in a relatively homogeneous population of patients [16]. However, dropout rates, which can impact negatively on interpretation of results in multi-cycle studies [13], in the pivotal trial were relatively high, ranging from 12 to $24 \%$, across cycles $2-4$. In our study, $98 \%$ of the patients completed

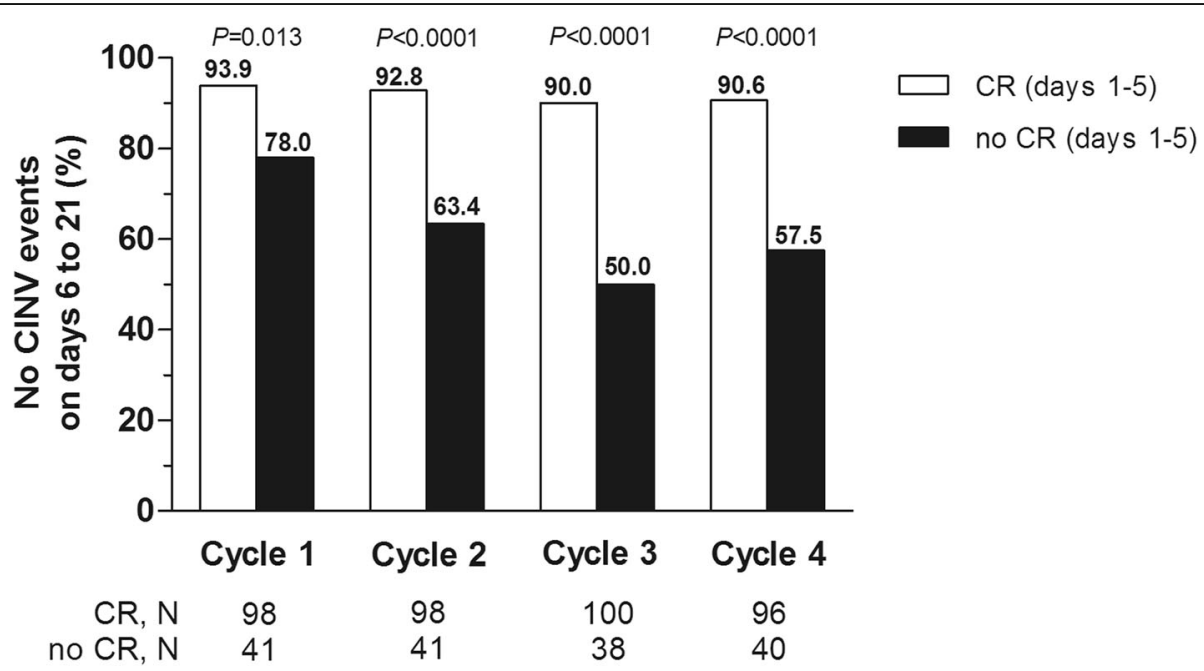

Fig. 2 Proportion of patients without CINV events during the very late period by CR status seen in the overall period of each cycle. CINV, chemotherapy-induced nausea and vomiting; CR, complete response (no emesis, and no use of rescue medication). $P$ values were calculated using the Fisher's exact test (two-sided). CINV events were vomiting and/or moderate-to-severe nausea 
Table 4 Multivariable regression analyses on predictors of CINV in the very late period (days 6 to 21) in patients with a CR during the overall observation period

\begin{tabular}{|c|c|c|c|}
\hline & Odds Ratioa & $95 \% \mathrm{Cl}$ & $\boldsymbol{P}$-value \\
\hline \multicolumn{4}{|l|}{ Cycle $1(n=98)$} \\
\hline Motion sickness & 1.50 & $0.21 ; 10.9$ & 0.687 \\
\hline Pregnancy-related morning sickness & 0.40 & $0.05 ; 3.00$ & 0.376 \\
\hline Alcohol intake (every day vs. none) & Not estimable & Not estimable & Not estimable \\
\hline Age ( $\geq 50$ vs. $<50$ years) & 0.69 & $0.10 ; 4.90$ & 0.707 \\
\hline Nausea over last 24 h (0-10 NRS) & 0.74 & $0.53 ; 1.03$ & 0.077 \\
\hline Anxiety over last 24 h (0-10 NRS) & 0.88 & $0.66 ; 1.17$ & 0.390 \\
\hline \multicolumn{4}{|l|}{ Cycle $2(n=98)$} \\
\hline Motion sickness & 1.50 & $0.25 ; 9.07$ & 0.660 \\
\hline Pregnancy-related morning sickness & 0.51 & $0.10 ; 2.70$ & 0.429 \\
\hline Alcohol intake (every day vs. none) & 1.05 & $0.10 ; 11.6$ & 0.968 \\
\hline Age ( $\geq 50$ vs. $<50$ years) & 0.30 & $0.05 ; 1.86$ & 0.197 \\
\hline Pre-chemotherapy nausea (0-10 NRS) & 0.97 & $0.64 ; 1.47$ & 0.870 \\
\hline Pre-chemotherapy anxiety (0-10 NRS) & 0.92 & $0.65 ; 1.29$ & 0.608 \\
\hline \multicolumn{4}{|l|}{ Cycle $3(n=100)$} \\
\hline Motion sickness & 0.44 & $0.10 ; 1.97$ & 0.281 \\
\hline Pregnancy-related morning sickness & 0.67 & $0.16 ; 2.87$ & 0.589 \\
\hline Alcohol intake (every day vs. none) & Not estimable & Not estimable & Not estimable \\
\hline Age ( $\geq 50$ vs. $<50$ years) & 2.16 & $0.47 ; 9.94$ & 0.323 \\
\hline Pre-chemotherapy nausea (0-10 NRS) & 0.65 & $0.50 ; 0.85$ & 0.001 \\
\hline Pre-chemotherapy anxiety (0-10 NRS) & 0.88 & $0.68 ; 1.15$ & 0.354 \\
\hline \multicolumn{4}{|l|}{ Cycle $4(n=96)$} \\
\hline Motion sickness & 0.11 & $0.01 ; 1.03$ & 0.053 \\
\hline Pregnancy-related morning sickness & 1.21 & $0.14 ; 10.6$ & 0.862 \\
\hline Alcohol intake (every day vs. none) & 0.77 & $0.04 ; 16.3$ & 0.868 \\
\hline Age ( $\geq 50$ vs. $<50$ years) & 0.11 & $0.01 ; 1.10$ & 0.061 \\
\hline Pre-chemotherapy nausea (0-10 NRS) & 0.50 & $0.34 ; 0.74$ & 0.0006 \\
\hline Pre-chemotherapy anxiety (0-10 NRS) & 1.16 & $0.78 ; 1.73$ & 0.470 \\
\hline
\end{tabular}

All patients received NEPA (netupitant/palonosetron) and dexamethasone on day 1 of each cycle; CINV chemotherapy-induced nausea and vomiting, CR complete response (no emesis and no use of rescue medication). Cl confidence interval, NRS numeric rating scale (a score of 1 or greater indicating the occurrence of symptoms)

aOdds ratio lower than 1 indicates an increased likelihood of developing CINV events during the very late period

the planned 4 cycles of AC-containing chemotherapy, and the very low dropout rate reinforces the clinical relevance of the study findings. More recently, in a registration trial that assessed the safety of intravenous NEPA compared to oral NEPA, both in combination with single-dose dexamethasone, in breast cancer patients treated with $\mathrm{AC}$, the proportion of patients receiving oral NEPA $(n=202)$ who achieved an overall CR ranged from 77 to $87 \%$ over cycles 1-4 [17]. However, only approximately $50 \%$ of patients completed all 4 cycles of treatment in this study.

In spite the advent of anti-emetics with novel mechanisms such as NK-1RAs, control of nausea still remains a clinical unmet need [18]. Since a very high-risk population for CINV was included in the current study, the rates of no significant nausea across cycles may be considered encouraging. The clinical benefit of NEPA against nausea over multiple cycles of $\mathrm{AC}$ is also supported by the previously mentioned pivotal trial where NEPA resulted in statistically significant superior rates of no significant nausea over palonosetron [16]. It is interesting to note that palonosetron plus dexamethasone was demonstrated to be superior to a first-generation $5-\mathrm{HT}_{3} \mathrm{RA}$ plus dexamethasone for the control of nausea in the setting of AC [19].

An additional benefit of the anti-emetic prophylaxis with NEPA is to provide an opportunity to overcome barriers interfering with guideline adherence in clinical practice. More recently, an observational, prospective 
study showed that the prevention of CINV caused by $\mathrm{AC}$ is suboptimal in Italian clinical practice, with prophylaxis with aprepitant during the delayed period being administered to less than half of 246 patients with breast cancer [20]. This finding is consistent with data from a previous European observational study evaluating the use of a guideline-consistent prophylaxis in patients receiving emetogenic chemotherapy regimens [21]. Last but not least, a single-day prophylaxis with NEPA may help to overcome non-adherence to medications such as prescribed delayed anti-emetics that was identified as a prevalent issue among patients with breast cancer, particularly younger patients, and may have a cumulative effect on the occurrence and severity of CINV [22]. In light of this, NEPA could improve adherence to guidelines by minimising the overall pill burden for patients who fear that the action of swallowing itself would induce nausea and vomiting [23].

This prospective study provided insight into the importance of achieving $C R$ in the overall period for the control of CINV events (i.e., vomiting and/or moderateto-severe nausea) during the very late period (day 6 through 21) in each cycle of AC. In an exploratory analysis, no CINV events in the very late period occurred in $94 \%$ of the patients with a CR in cycle 1 , with slightly lower incidence in later cycles of chemotherapy. In the subgroup of patients without a $C R$, significantly fewer patients were free from CINV events over the very late period in all chemotherapy cycles. These findings suggest that $\mathrm{CR}$ over the 5-day period of highest emetic risk after $\mathrm{AC}$ administration also plays an important part in the prevention of CINV over the very late period in each cycle. Among the patients with a $\mathrm{CR}$, the predictive value of well-known risk factors for CINV was examined in a multivariable analysis for each cycle of AC. The only independent factor that negatively impacted the control of very late CINV was pre-chemotherapy nausea, with an effect observed from cycle 3 onwards. Prechemotherapy (anticipatory) nausea is a known risk factor for CINV, and a number of factors can place patients at higher risk of pre-chemotherapy nausea, including age, experiencing CINV in previous cycle, motion sickness, and female sex [12, 24]. Recently, a large, prospective observational study showed that pre-chemotherapy nausea is a predictor of $\mathrm{CR}$ in the acute, delayed, and overall periods alongside the use of guideline-consistent prophylaxis, younger age, and incomplete CINV response in an earlier cycle [6]. It should be noted that $21 \%$ of the patients in our study reported a score of 1 or greater for nausea on NRS just before receiving the first cycle of chemotherapy. Although this was also seen in recent, prospective, observational studies [12, 24], the clinical implications of this evidence still remain to be understood in CINV research.

\section{Conclusion}

The results of this study indicate that the high antiemetic efficacy seen with the NEPA regimen in the first cycle was maintained over multiple cycles of AC for breast cancer. Preliminary evidence suggests that the achievement of a $\mathrm{CR}$ in the overall period impacts also the risk for very late CINV in each cycle of AC. Therefore, CINV prevention over the 5-day period after chemotherapy administration remains a goal to improve control of symptoms for the whole duration of treatment cycle. As a single dose of NEPA and dexamethasone offers both effective and convenient guideline-consistent prophylaxis, future studies are warranted to determine the most feasible anti-emetic strategy to maximally prevent the nausea component of CINV over the entire risk period for each cycle of $\mathrm{AC}$ chemotherapy.

\section{Abbreviations \\ AC: Anthracycline and cyclophosphamide; AE: Adverse event; CINV: Chemotherapy-induced nausea and vomiting; CR: Complete response; CTCAE: Common terminology criteria for adverse events; 5- $\mathrm{HT}_{3} \mathrm{RA}$ : 5- hydroxytryptamine-3 receptor antagonist; HEC: Highly emetogenic chemotherapy; NK-1RA: Neurokinin-1 receptor antagonist; NRS: Numerical rating scale; TEAE: Treatment-emergent adverse event}

\section{Acknowledgements \\ We thank the clinical investigators, patients, study nurses, data management associates and site personnel who participated in the study. We also thank Italfarmaco S.p.A., Milan, Italy, for kindly providing the study drug.

\begin{abstract}
Authors' contributions
Conception and design: MDL, RC, SDP. Provision of study material or patients: all enrolling investigators. Data analysis and interpretation: RC, MEC, $A S, R T, I P, M G, V M, V G, L A, D B, G C, A F, E C, A S, E B, L C, S D P, M D L$. Manuscript writing: LC, MDL, RC. Final approval of manuscript: RC, MEC, AS, RT, IP, MG, $V M, V G, L A, D B, G C, A F, E C, A S, E B, L C, S D P, M D L$. Accountable for all aspects of the work: MDL, RC, SDP. The authors read and approved the final
\end{abstract} manuscript.}

\section{Funding}

This research did not receive any specific grant from funding agencies in the public, commercial, or not-for-profit sectors.

\section{Availability of data and materials}

The datasets used during the current study are available from the corresponding author on reasonable request.

\section{Ethics approval and consent to participate}

This study was approved by the Institutional Review Board of the National Cancer Institute "Fondazione Pascale", Naples (IRB number 70/15), and this trial is registered with Clinicaltrials.gov identifier, NCT03862144. All participants participated voluntarily and completed a written informed consent.

\section{Consent for publication}

Not applicable.

\section{Competing interests}

LC: fees for advisory board (Italfarmaco, Kyowa). MDL: advisory board e SC member (Novartis; Amgen; Eli Lilly; Genentech). VG: institutional research grant (Roche), fees for advisory board (Eli Lilly, Novartis, Roche), speakers bureau (Eli Lilly, Novartis).

IP: advisory board (Novartis, Roche, Astrazeneca, Eisai, Italfarmaco, Pfizer, Eli Lilly, Pierre-Fabre), invited speech (Novartis, Roche, Astrazeneca, Pfizer, Eli Lilly). 
SDP: advisory board (GSK, Novartis, Roche, Celgene, Astrazeneca, Amgen, Eisai, Italfarmaco, Pfizer, Eli Lilly), invited speech (GSK, Novartis, Roche, Celgene, Astrazeneca, Amgen, Pfizer, Eli Lilly).

RC: advisory board (Novartis, Italfarmaco).

Other authors declare that they have no competing interests.

\section{Author details}

${ }^{1}$ Breast Medical Oncology Division, Istituto Nazionale Tumori IRCCS "Fondazione G. Pascale", Naples, Italy. ${ }^{2}$ Medical Oncology Unit \& Phase 1 Research Unit, ASST Monza, Monza, Italy. ${ }^{3}$ Medical Oncology Unit, Department of Translational Research and New Technologies in Medicine and Surgery, University of Pisa, Pisa, Italy. ${ }^{4}$ Department of Medical Oncology and Hematology, Humanitas Clinical and Research Center, Rozzano, Milan, Italy. ${ }^{5}$ Division of Gynecologic Oncology, Fondazione Policlinico Universitario A. Gemelli IRCCS, Rome, Italy. ${ }^{6}$ Medical Oncology Division, ASST-Lariana, Como, Italy. ${ }^{7}$ Oncology Unit, "Monaldi" Hospital, Naples, Italy. ${ }^{8}$ Department of Surgery, Oncology and Gastroenterology, University of Padova, Padova, Italy. ${ }^{9}$ Istituto Oncologico Veneto IOV I.R.C.C.S, Padova, Italy. ${ }^{10}$ Oncology Department Area Vasta Romagna, Faenza Hospital, Faenza, Ravenna, Italy. ${ }^{11}$ Medical Oncology Unit, S. Carlo Hospital, Potenza, Italy. ${ }^{12}$ Medical Oncology Division, Fondazione IRCCS Casa Sollievo Della Sofferenza, San Giovanni Rotondo, Foggia, Italy. ${ }^{13}$ IRCCS Regina Elena National Cancer Institute, Rome, Italy. ${ }^{14}$ Oncology Unit, ASST Ovest Milanese, Legnano Hospital, Legnano, Milan, Italy. ${ }^{15}$ Clinical Oncology Division, Azienda Ospedaliero-Universitaria, Cona, Ferrara, Italy. ${ }^{16}$ Department of Clinical Science and Community. Section of Medical Statistics, Biometry and Epidemiology "G.A. Maccacaro". Faculty of Medicine and Surgery, University of Milan, Milan, Italy. ${ }^{17}$ Medical Oncology Unit 1, Fondazione IRCCS "Istituto Nazionale dei Tumori", Milan, Italy. ${ }^{18}$ Clinical Medicine and Surgery Department, University of Naples Federico II, Naples, Italy.

Received: 12 July 2019 Accepted: 3 March 2020

Published online: 19 March 2020

\section{References}

1. Navari RM, Aapro M. Antiemetic prophylaxis for chemotherapy-induced nausea and vomiting. N Engl J Med. 2016;374:1356-67.

2. Warr DG, Hesketh PJ, Gralla RJ, Muss HB, Herrstedt J, Eisenberg PD, et al. Efficacy and tolerability of aprepitant for the prevention of chemotherapyinduced nausea and vomiting in patients with breast cancer after moderately emetogenic chemotherapy. J Clin Oncol. 2005;23:2822-30.

3. Bash E, Prestrud AA, Hesketh PJ, Kris MG, Feyer PC, Somerfield MR, et al. Antiemetics: American Society of Clinical Oncology clinical practice guideline update. J Clin Oncol. 2011;29:4189-98.

4. Roila F, Molassiotis A, Herrstedt J, Aapro M, Gralla RJ, Bruera E, et al. 2016 MASCC and ESMO guideline update for the prevention of chemotherapyand radiotherapy-induced nausea and vomiting and of nausea and vomiting in advanced cancer patients. Ann Oncol. 2016;27(Suppl 5):v11933.

5. Hesketh PJ, Kris MG, Basch E, Bohlke K, Barbour SY, Clark-Snow RA, et al. Antiemetics: American Society of Clinical Oncology clinical practice guideline update. J Clin Oncol. 2017;35:3240-61.

6. Molassiotis A, Aapro M, Dicato M, Gascon P, Novoa SA, Isambert N, et al. Evaluation of risk factors predicting chemotherapy-related nausea and vomiting: results from a European prospective observational study. J Pain Symptom Manage. 2014:47:839-48.

7. Hesketh PJ, Aapro M, Jordan K, Schwartzberg L, Bosnjak S, Rugo H. A review of NEPA, a novel fixed antiemetic combination with the potential of enhancing guideline adherence and improving control of chemotherapyinduced nausea and vomiting. Biomed Res Int. 2015;2015:651879.

8. Rojas C, Mithun R, Tsukamoto T, Slusher BS. Molecular mechanisms of 5-HT and $\mathrm{NK}_{1}$ receptor antagonists. Eur J Pharmacol. 2014;722:26-37.

9. Celio L, Niger M, Ricchini F, Agustoni F. Palonosetron in the prevention of chemotherapy-induced nausea and vomiting: an evidence-based review of safety, efficacy, and place in therapy. Core Evid. 2015;10:75-87.

10. Aapro M, Rugo H, Rossi G, Rizzi G, Borroni ME, Bondarenko I, et al. A randomized phase III study evaluating the efficacy and safety of NEPA, a fixed-dose combination of netupitant and palonosetron, for prevention of chemotherapy-induced nausea and vomiting following moderately emetogenic chemotherapy. Ann Oncol. 2014;25:1328-33.
11. Spinelli T, Moresino C, Baumann S, Timmer W, Schultz A. Effects of combined netupitant and palonosetron (NEPA), a cancer supportive care antiemetic, on the ECG of healthy subjects: an ICH E14 thorough QT trial. Springerplus. 2014;3:389.

12. Chan A, Kim H-K, Hsieh RK, Yu S, de Lima LG, Su W-C, et al. Incidence and predictors of anticipatory nausea and vomiting in Asia Pacific clinical practice-a longitudinal analysis. Support Care Cancer. 2015;23:283-91.

13. Herrstedt J, Muss HB, Warr DG, Hesketh PJ, Eisenberg PD, Raftopoulos H, et al. Efficacy and tolerability of aprepitant for the prevention of chemotherapy-induced nausea and emesis over multiple cycles of moderately emetogenic chemotherapy. Cancer. 2005;104:1548-55.

14. Shih V, Wan HS, Chan A. Clinical predictors of chemotherapy-induced nausea and vomiting in breast cancer patients receiving adjuvant doxorubicin and cyclophosphamide. Ann Pharmacother. 2009:43:444-52.

15. Warr DG, Street JC, Carides AD. Evaluation of risk factors predictive of nausea and vomiting with current standard-of-care antiemetic treatment: analysis of phase 3 trial of aprepitant in patients receiving adriamycincyclophosphamide-based chemotherapy. Support Care Cancer. 2011;19: 807-13.

16. Aapro M, Karthaus M, Schwartzberg L, Bondarenko I, Sarosiek T, Oprean C, et al. NEPA, a fixed oral combination of netupitant and palonosetron, improves control of chemotherapy-induced nausea and vomiting (CINV) over multiple cycles of chemotherapy: results of a randomized, doubleblind, phase 3 trial versus oral palonosetron. Support Care Cancer. 2017;25: 1127-35.

17. Schwartzberg L, Navari R, Clark-Snow R, Arkania E, Radyukova I, Patel K, et al. Phase IIlb safety and efficacy of intravenous NEPA for prevention of chemotherapy-induced nausea and vomiting (CINV) in patients with breast cancer receiving initial and repeat cycles of anthracycline and cyclophosphamide (AC) chemotherapy. Oncologist. 2019. https://doi.org/10. 1634/theoncologist.2019-0527.

18. Bosnjak SM, Gralla RJ, Schwartzberg L. Prevention of chemotherapy-induced nausea: the role of neurokinin-1 $\left(\mathrm{NK}_{1}\right)$ receptor antagonists. Support Care Cancer. 2017;25:1661-71.

19. Kubota K, Saito M, Aogi K, Sekine I, Yoshizawa H, Yanagita Y, et al. Control of nausea with palonosetron versus granisetron, both combined with dexamethasone, in patients receiving cisplatin or anthracycline plus cyclophosphamide-based regimens. Support Care Cancer. 2016;24:4025-33.

20. De Laurentiis M, Bonfadini C, Lorusso V, Cilenti G, Di Rella F, Altavilla G, et al. Incidence of nausea and vomiting in breast cancer patients treated with anthracycline plus cyclophosphamide-based chemotherapy regimens in Italy: NAVY observational study. Support Care Cancer. 2018. https://doi.org/ 10.1007/s00520-018-4259-1.

21. Aapro M, Molassiotis A, Dicato M, Pelaez I, Rodriguez-Lescure A, Pastorelli D, et al. The effect of guideline-consistent antiemetic therapy on chemotherapy-induced nausea and vomiting (CINV): the Pan European Emesis registry (PEER). Ann Oncol. 2012;23:1986-92.

22. Chan A, Low XH, Yap KY. Assessment of the relationship between adherence with antiemetic drug therapy and control of nausea and vomiting in breast cancer patients receiving anthracycline-based chemotherapy. J Manag Care Pharm. 2012;18:385-94.

23. Vidall C, Fernandez-Ortega P, Cortinovis D, Jahan P, Amlani B, Scottè F. Impact and management of chemotherapy/radiotherapy-induced nausea and vomiting and the perceptual gap between oncologists/oncology nurses and patients: a cross-sectional multinational survey. Support Care Cancer. 2015;23:3297-305.

24. Molassiotis A, Lee PH, Burke TA, Dicato M, Gascon P, Roila F, et al. Anticipatory nausea, risk factors, and its impact on chemotherapy-induced nausea and vomiting: results from the Pan European Emesis registry study. J Pain Symptom Manag. 2016;51:987-93.

\section{Publisher's Note}

Springer Nature remains neutral with regard to jurisdictional claims in published maps and institutional affiliations. 